\title{
Effect of Primary User Traffic on Largest Eigenvalue Based Spectrum Sensing Technique
}

\author{
Pawan Dhakal $^{1}$, Shree K. Sharma ${ }^{2}$ Symeon Chatzinotas ${ }^{2}$, Björn Ottersten ${ }^{2}$, \\ and Daniel Riviello ${ }^{1}$ \\ 1 Politecnico di Torino, Torino, Italy \\ \{pawan.dhakal, daniel.riviello\}@polito.it \\ 2 University of Luxembourg, SnT, Kirchberg, Luxembourg \\ \{shree.sharma, symeon.chatzinotas, bjorn.ottersten\}@uni.lu
}

\begin{abstract}
In this paper, the effect of primary user (PU) traffic on the performance of largest eigenvalue based spectrum sensing technique (Roy's Largest Root Test (RLRT)) is investigated. A simple and realistic discrete time modeling of PU traffic is considered which is only based on the discrete time distribution of PU free and busy periods. Furthermore, in order to analyze the effect of PU traffic on the detection performance, analytical expressions for the probability density functions of the decision statistic are derived and validated by Monte-Carlo simulations. Numerical results demonstrate that the sensing performance of RLRT is no more monotonically increasing with the length of the sensing duration and also with SNR which contrasts with the common property of the spectrum sensing techniques under known PU traffic scenario. Furthermore, it is shown that the performance gain due to the multiple antennas in the sensing unit is significantly suppressed by the effect of the PU traffic when the frequency of the PU traffic transitions is higher.
\end{abstract}

Key words: Eigenvalue based detection, Cognitive radio, Spectrum sensing, RLRT, Primary user traffic

\section{Introduction}

By accessing the idle spectrum band of Primary User (PU) network (licensed user), Cognitive Radio (CR) based dynamic spectrum sharing is initially intended to alleviate one of the most challenging problems of future wireless communications, namely, spectrum scarcity. With the real-time perception of surroundings and bandwidth availability using spectrum sensing functionality of a CR, secondary users (unlicensed users) may dynamically use the vacant spectrum and perform opportunistic transmissions[1]. Thus, the domain of spectrum sensing techniques has long been investigated by many researchers: a detailed bibliography of the contributions in this area can be found in $[2,3]$. Despite the significant volume of available literature on spectrum sensing under ideal scenarios, investigation under practical constraints and imperfections are still lacking [3]. Thus, recent research efforts are devoted to improve the accuracy 
and efficiency of sensing techniques under practical constraints and imperfections.

Among many practical imperfections and constraints for spectrum sensing in $\mathrm{CR}$ scenarios mentioned in the literature, the unknown PU traffic is one of the important constraints which significantly limits the sensing performance of the secondary user. In the existing literature on spectrum sensing, the SUs are assumed to have a perfect knowledge of the exact time slot structure of PU transmissions providing a solid basis for guaranteeing that PU traffic transitions occur only at the beginning of the SU sensing slots. However, in practice, the SU may not have the knowledge of exact time slot structure of PU transmissions or it is also possible that the communications among PUs are not based on synchronous schemes at all $[4,5]$. Thus, it is necessary to analyze the sensing performance of existing spectrum sensing techniques under unknown PU traffic.

A first attempt to analyze the performance of a detector in unknown PU traffic was made in [6]. The author analyzed the sensing performance of the well known semi-blind spectrum sensing techniques including Energy Detection (ED) and Roy's Largest Root Test (RLRT) under bursty PU traffic. The PU traffic model used in [6] is limited to a constant burst length of the PU data whose length is assumed to be always shorter than the SU sensing duration. However, the burst length of the PU may vary with time following some stochastic models $[7,8]$. A more general scenario in which the PU traffic transitions are completely random has been considered in $[9,10,11,12,13]$. By modeling PU traffic as a two state Markov process, authors in $[9,10,11,12]$ analyzed the effect of PU traffic on the sensing performance and the sensing-throughput trade-off considering an ED technique under the half duplex scenario. Moreover, the effect of multiple PUs traffic on the sensing-throughput trade-off of the secondary system has been studied in [13]. Although all the aforementioned contributions recognized the fact that the PU traffic affects the sensing performance including sensing-throughput trade-off, none of them analyzed the sensing performance of other spectrum sensing techniques including Eigenvalue Based Detection (EBD) techniques under unknown primary user traffic.

In this paper, the effect of PU traffic on the performance of RLRT is evaluated. First, a realistic discrete time modeling of PU traffic is considered which is only based on the discrete time distribution of PU free and busy periods. Next, the analytical expressions for the probability density functions (pdfs) of the decision statistic are derived and validated by Monte-Carlo simulations. Finally, an analytical performance evaluation of the decision statistic in terms of receiver operating characteristics (ROC) under the considered scenario is carried out.

\section{System Model}

We consider a single source scenario (single primary transmitter) whereas multiple antennas are employed by an SU. Suppose the SU has $K$ antennas and each antenna receives $\mathrm{N}$ samples in each sensing slot. In a given sensing frame, the detector calculates its decision statistic $T_{D}$ by collecting $N$ samples from 
each one of the $K$ antennas. Subsequently, the received samples are collected by the detector in the form of a $K \times N$ matrix $\mathbf{Y}$. As described in Sec. 1, when the primary transmissions are not based on some synchronous schemes or the sensing unit at the SU does not have any information about the primary traffic pattern, the received vector at the sensing unit may consist of partly the samples from one PU state and the remaining from alternate PU state. To simplify the scenario, we begin with the following classification of the sensing slots based on the PU traffic status, which is also illustrated in Fig. 1.

1. Steady State (SS) sensing slot: In such type of sensing slot, all the received samples in one sensing slot are obtained from the same PU state.

2. Transient State (TS) sensing slot: In such type of sensing slot, a part of the received samples within the sensing slot are obtained from one PU state and the remaining from the another PU state.

In general, the probabilities of receiving SS and TS sensing slots are dependent on the PUs traffic model. In contrast to the commonly used hypothesis definition in spectrum sensing literature, we define two hypotheses in the following way:

$\mathcal{H}_{0}$ : the channel is going to be free,

$\mathcal{H}_{1}$ : the channel is going to be busy.

This hypothesis formulation implies that the decision is based on the PU status at the end of the sensing interval. Thus, in a TS sensing slot, a transition from the PU busy state to the PU free state is considered $\mathcal{H}_{0}$, while a transition from the PU free state to the PU busy state is considered $\mathcal{H}_{1}$. In the considered scenario, in an SS sensing interval, the generic received signal matrix under each hypothesis can be written as,

$$
\boldsymbol{Y}_{S S}= \begin{cases}\boldsymbol{V}_{[K, N]} & \left(\mathcal{H}_{0}\right), \\ \boldsymbol{S}_{[K, N]} & \left(\mathcal{H}_{1}\right),\end{cases}
$$

where $\boldsymbol{V}_{[K, N]} \triangleq[\boldsymbol{v}(1) \cdots \boldsymbol{v}(n) \cdots \boldsymbol{v}(N)]$ is the $K \times N$ noise matrix, $\boldsymbol{S}_{[K, N]}=$ $\mathbf{h}_{[K, 1]} \mathbf{S}_{[1, N]}+\mathbf{V}_{[K, N]}$ is the $K \times N$ received noisy signal matrix when PU signal is present. $\boldsymbol{h}_{[K, 1]}=\left[h_{1} \cdots h_{K}\right]^{T}$ is the channel vector and $\boldsymbol{s}_{[1, N]} \triangleq[s(1) \cdots s(n) \cdots s(N)]$ is a $1 \times N$ PU signal vector. And in the TS sensing interval, the generic received signal matrix under each hypothesis can be written as,

$$
\boldsymbol{Y}_{T S}=\left\{\begin{array}{l}
{\left[\mathbf{S}_{\left[K, N-D_{0}\right]} \mid \mathbf{V}_{\left[K, D_{0}\right]}\right]\left(\mathcal{H}_{0}\right),} \\
{\left[\mathbf{V}_{\left[K, N-D_{1}\right]} \mid \mathbf{S}_{\left[K, D_{1}\right]}\right]\left(\mathcal{H}_{1}\right),}
\end{array}\right.
$$

where $D_{0}$ represents the number of pure noise samples in TS sensing slot under $\mathcal{H}_{0}, D_{1}$ represents the number of noise plus PU signal samples in TS sensing slot under $\mathcal{H}_{1}, \mathbf{S}_{\left[K, N-D_{0}\right]}=\mathbf{h}_{[K, 1]} \mathbf{S}_{\left[1, N-D_{0}\right]}+\mathbf{V}_{\left[K, N-D_{0}\right]}$ is the $\left(K \times N-D_{0}\right)$ received noisy signal matrix when $\mathrm{PU}$ signal is present only for $\left(N-D_{0}\right)$ sample periods. Similarly, $\mathbf{S}_{\left[K, D_{1}\right]}=\mathbf{h}_{[K, 1]} \mathbf{S}_{\left[1, D_{1}\right]}+\mathbf{V}_{\left[K, D_{1}\right]}$ is the $K \times D_{1}$ received noisy signal matrix when PU signal is present only for $D_{1}$ sample periods. In each of these, the unknown primary transmitted signal $s(n)$ at time instant $n$ is modeled as independent and identically distributed (i.i.d.) complex Gaussian with zero 


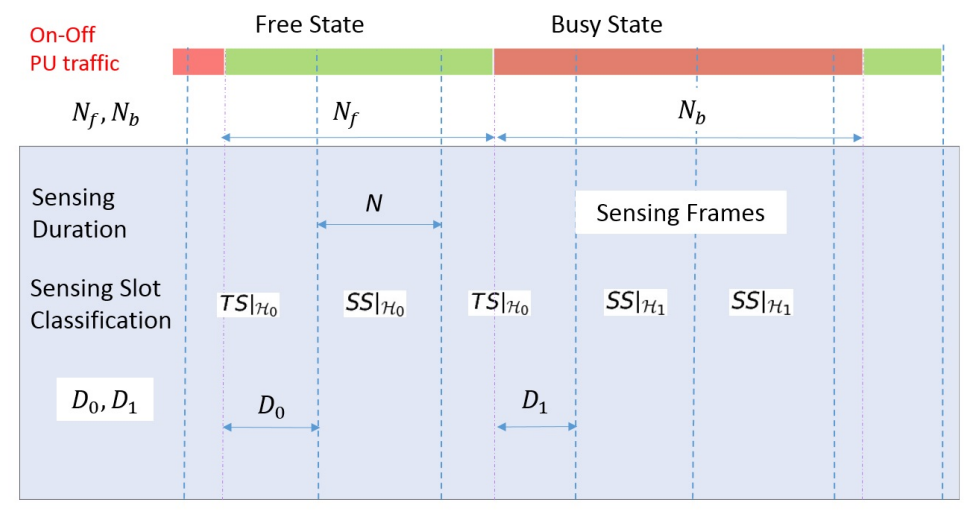

Fig. 1: Primary user traffic scenario and sensing slot classification

mean and variance $\sigma_{s}^{2}: s(n) \sim \mathcal{N}_{\mathbb{C}}\left(0, \sigma_{s}^{2}\right)$. The noise sample $v_{k}(n)$ at the $k^{t h}$ antenna of the SU at the time instant $n$ is also modeled as complex Gaussian with mean zero and variance $\sigma_{v}^{2}: v_{k}(n) \sim \mathcal{N}_{\mathbb{C}}\left(0, \sigma_{v}^{2}\right)$. The channel coefficient $h_{k}$ of the $k^{t h}$ antenna is assumed to be constant and memory-less during the sensing interval. The average SNR at the receiver is defined as, $\rho=\frac{\sigma_{s}^{2}\|\boldsymbol{h}\|^{2}}{K \sigma_{v}^{2}}$, where $\|\cdot\|$ denotes the Euclidean norm.

\section{Characterization of Primary User Traffic}

In this section, we characterize the mathematical model of PU traffic . Based on the proposed stochastic PU traffic model, we construct the PU's probability transition matrix, which lead to analytical formulation of the SU's probability of receiving SS sensing frame and TS sensing frame under each null and alternate hypothesis.

In this paper, we model the PU traffic as a two state Markovs process (OnOff process: $\mathrm{PU}$ 'On' representing busy state and $\mathrm{PU}$ 'Off' representing free state). The length of free as well as busy period are independent geometrically distributed random variables with parameters $\alpha$ and $\beta$, respectively. Essentially, the parameters $\alpha$ and $\beta$ represent the state transition probabilities in single sample duration. The mean length of free period $M_{f}$ and busy period $M_{b}$ of $\mathrm{PU}$ traffic can be related to parameters $\alpha$ and $\beta$ as, $M_{f}=\frac{1}{\alpha}$ and $M_{b}=\frac{1}{\beta}$, respectively.

At any time instant, the $\mathrm{PU}$ is in free state with probability $P_{f}=\frac{M_{f}}{M_{b}+M_{f}}$ and similarly, in the busy state with probability, $P_{b}=\frac{M_{b}}{M_{b}+M_{f}}$. We further assume that the parameters ( $\alpha$ and $\beta$ ) of geometrically distributed length of PU free and busy periods are constant over time. Thus, the corresponding two-state Markovs process can be considered homogeneous in nature. Using this homo- 
geneity property and the Chapman-Kolmogorov equation gives the PU n-step transition probability matrix as,

$$
\begin{aligned}
& \mathbf{P}^{n}=\left[\begin{array}{ll}
p_{00}^{n} & p_{01}^{n} \\
p_{10}^{n} & p_{11}^{n}
\end{array}\right] \\
& =\frac{1}{\alpha+\beta}\left[\begin{array}{cc}
\beta+\alpha(1-\alpha-\beta)^{n} & \alpha-\alpha(1-\alpha-\beta)^{n} \\
\beta-\beta(1-\alpha-\beta)^{n} & \alpha+\beta(1-\alpha-\beta)^{n}
\end{array}\right],
\end{aligned}
$$

which reduces to (4) for single step transition matrix as,

$$
\mathbf{P}=\left[\begin{array}{ll}
p_{00} & p_{01} \\
p_{10} & p_{11}
\end{array}\right]=\left[\begin{array}{cc}
1-\alpha & \alpha \\
\beta & 1-\beta
\end{array}\right]
$$

As already mentioned earlier in Sec. 2, the stochastic nature of the PU state transition gives a mixed nature of received signals in a TS sensing slot resulting in random variables (RVs) $D_{0}$ and $D_{1}$. Thus, in each PU state transition from Busy to Free State, the sensing unit has to decide based on $D_{0}$ pure noise samples and $\left(N-D_{0}\right)$ noise plus primary signal samples which actually affects the overall sensing performance. Thus, with the support of above analysis and also keeping (1) and (2) in reference, it is clear that, to find the distribution of the decision statistic under different hypotheses, the prior deduction of the chances of occurrence of SS sensing slot, TS sensing slot, probability mass function (pmf) of $D_{0}$ and the pmf of $D_{1}$ are inevitable. It can be shown that ${ }^{1}$, under the assumption that the lengths of busy period and free period of $\mathrm{PU}$ have comparable mean parameters $M_{f}$ and $M_{b}$, the pmf of $D_{0}$ which represents the probability of having $D_{0}$ noise only (PU signal free) samples in a TS sensing slot under $\mathcal{H}_{0}$ reduces to,

$$
P_{D_{0}}\left(D_{0}=d_{0}\right)= \begin{cases}\frac{1}{N-1} & 1 \leq d_{0}<N, \\ 0 & \text { otherwise. }\end{cases}
$$

Similarly, the pmf of $D_{1}$ which represents the probability of having $D_{1}$ PUsignal-plus-noise samples in a TS sensing slot under $\mathcal{H}_{1}$ reduces to,

$$
P_{D_{1}}\left(D_{1}=d_{1}\right)= \begin{cases}\frac{1}{N-1} & 1 \leq d_{1}<N, \\ 0 & \text { otherwise. }\end{cases}
$$

Also, the probability of receiving SS sensing slot under $\mathcal{H}_{0}$ is given by,

$$
\left.P_{S S}\right|_{\mathcal{H}_{0}}=\frac{1}{1+\frac{N}{M_{f}}} .
$$

and the probability of receiving SS sensing slot under $\mathcal{H}_{1}$ is given by,

$$
\left.P_{S S}\right|_{\mathcal{H}_{1}}=\frac{1}{1+\frac{N}{M_{b}}} .
$$

\footnotetext{
${ }^{1}$ Due to space limitation, we omit the proofs of following equations and will include in the journal version of the paper.
} 


\section{Sensing Performance Analysis}

In this section, we consider an another important class of detection techniques, designed for multi-sensor detectors, based on the eigenvalues of the received signal covariance matrix. Receive diversity can be achieved either by multiple users (cooperative detection) or by multiple antennas. Given a $K \times N$ received signal matrix $\mathbf{Y}$, the sample covariance matrix is defined as, $\mathbf{R} \triangleq \frac{1}{N} \mathbf{Y} \mathbf{Y}^{\mathrm{H}}$ and $\lambda_{1} \geq \cdots \geq \lambda_{k}$ its eigenvalues sorted in the decreasing order.

Eigenvalue based detection techniques infer the presence of signal from eigenvalues $\lambda_{i}$. In particular, the detection technique which considers the largest one $\left(\lambda_{1}\right)$ and compare against the noise variance is known in statistics as Roy's Largest Root Test (RLRT) [15] and its test statistics is,

$$
T_{R L R T} \triangleq \frac{\lambda_{1}}{\sigma_{v}^{2}}
$$

RLRT is "semi-blind" as it requires the exact knowledge of noise variance and is considered to be asymptotically optimum test in this setting[17]. Other related tests have been proposed in the literature for example $\lambda_{1}$ against smallest eigenvalue [16], $\lambda_{1}$ against trace of covariance matrix [18]. These are considered "blind" as they do not require the prior knowledge of the noise variance.

Here, we analyze in detail the RLRT method. However, the results can be extended to the other methods as well. To analyze the RLRT performance, it is necessary to express the pdf of the test statistics for the case of unknown PU traffic. The following theorem computes the pdf of the RLRT decision statistic under both the hypotheses using the PU traffic characterization presented in Sec. 3 .

Theorem 1. Given a multi-antenna sensing unit with $K$ receive antennas, $N$ received samples in each slot and the random $P U$ traffic with geometrically distributed free and busy state duration, let $c=K / N, N_{s}$ a independent parameter and define:

$$
\begin{aligned}
\mu_{1}\left(N_{s}\right) & =\left(\frac{N_{s}}{N} K \rho+1\right)\left(1+\frac{K-1}{N_{s} K \rho}\right), \quad \sigma_{1}^{2}\left(N_{s}\right)=\frac{N_{s}}{N^{2}}(K \rho+1)\left(1-\frac{K-1}{N_{s} K^{2} \rho^{2}}\right)(10) \\
\mu_{N, K} & =[1+\sqrt{c}]^{2}, \quad \sigma_{N, K}=N^{-2 / 3}[1+\sqrt{c}]\left[1+\frac{1}{\sqrt{c}}\right]^{1 / 3}
\end{aligned}
$$

Then, the pdfs of RLRT decision statistic under $\mathcal{H}_{0}$ and $\mathcal{H}_{1}$ are given by $(12)$ and (13) respectively,

$$
\begin{aligned}
& f_{\left.T_{R L R T}\right|_{H_{0}}}(x)=\left.P_{S S}\right|_{H_{0}} f_{T W 2}\left(\frac{x-\mu_{N, K}}{\sigma_{N, K}}\right)+\left.P_{T S}\right|_{H_{0}} \sum_{d_{0}=1}^{N-1} P_{D_{0}}\left(d_{0}\right) f_{D}\left(x, N-d_{0}\right), \\
& f_{\left.T_{R L R T}\right|_{H_{1}}}(x)=\left.P_{S S}\right|_{\mathcal{H}_{1}} f_{D}(x, N)+\left.P_{T S}\right|_{\mathcal{H}_{1}} \sum_{d_{1}=1}^{N-1} P_{D_{1}}\left(d_{1}\right) f_{D}\left(x, d_{1}\right) .
\end{aligned}
$$

where,

$$
f_{D}(x, d)= \begin{cases}f_{\mathcal{N}}\left(\mu_{1}(d), \sigma_{1}^{2}(d)\right) & \text { if, } d>\frac{K-1}{K^{2} \rho^{2}}, \\ f_{T W 2}\left(\mu_{N, K}, \sigma_{N, K}\right) & \text { otherwise, }\end{cases}
$$


In (14), $f_{\mathcal{N}}\left(\mu_{1}(d), \sigma_{1}^{2}(d)\right)$ denote a Gaussian pdf with mean $\mu_{1}\left(N_{s}\right)$ and variance $\sigma_{1}^{2}\left(N_{s}\right)$ provided in $(10)$ at $N_{s}=d$. Next, $f_{T W 2}\left(\mu_{N, K}, \sigma_{N, K}\right)$ is a pdf of TracyWidom distribution of order 2 with parameters $\mu_{N, K}$ and $\sigma_{N, K}$ provided in (11).

Proof. As noted from Sec. 2, the nature of the received signal matrix is different for the SS sensing slot and TS sensing slot. Under null hypothesis $\mathcal{H}_{0}$, received sample covariance matrix $\left.\mathbf{R}\right|_{\mathcal{H}_{0}}$ can be decomposed as a probabilistic sum of $\left.\mathbf{R}_{S S}\right|_{\mathcal{H}_{0}}$ and $\left.\mathbf{R}_{T S}\right|_{\mathcal{H}_{0}}$ in the following way,

$$
\left.\mathbf{R}\right|_{\mathcal{H}_{0}}=\left.\left.P_{S S}\right|_{\mathcal{H}_{0}} \mathbf{R}_{S S}\right|_{\mathcal{H}_{0}}+\left.\left.P_{T S}\right|_{\mathcal{H}_{0}} \mathbf{R}_{T S}\right|_{\mathcal{H}_{0}} .
$$

Since sensing slots are independent from each other, we treat each covariance matrix in (15) independently. Given an SS sensing slot under null hypothesis, all the received samples $y_{k}(n)$ are homogeneous in nature comprising the i.i.d. Gaussian noise samples with mean zero and variance $\sigma_{v}^{2}$. Thus, the sample covariance matrix $\left.\mathbf{R}_{S S}\right|_{\mathcal{H}_{0}}$ follows a Wishart distribution whose largest eigenvalue normalized by noise variance can be expressed by a Tracy-Widom distribution of second order $[17,20]$.

$$
\frac{\left.\lambda_{1}^{S S}\right|_{\mathcal{H}_{0}}}{\sigma_{v}^{2}}=f_{T W 2}\left(\frac{x-\mu_{N, K}}{\sigma_{N, K}}\right)
$$

where $\mu_{N, K}$ and $\sigma_{N, K}$ are given in (11).

Next, given a TS sensing slot under null hypothesis, all the received samples $y_{k}(n)$ are not homogeneous in nature. To provide a better understanding, we express the covariance matrix in a TS sensing slot under $\mathcal{H}_{0}$ as,

$$
\left.\mathbf{R}_{T S}\right|_{\mathcal{H}_{0}}=\mathbf{R}_{S}\left(N-D_{0}\right)+\mathbf{R}_{N}\left(D_{0}\right),
$$

where,

$$
\begin{aligned}
\mathbf{R}_{S}\left(N-D_{0}\right) & \triangleq \frac{1}{N-D_{0}} \mathbf{S}_{\left[K, N-D_{0}\right]} \mathbf{S}_{\left[K, N-D_{0}\right]}^{H}, \\
\mathbf{R}_{N}\left(D_{0}\right) & \triangleq \frac{1}{D_{0}} \mathbf{V}_{\left[K, D_{0}\right]} \mathbf{V}_{\left[K, D_{0}\right]}^{H},
\end{aligned}
$$

are the partial covariance matrices constructed respectively from signal-plusnoise and only-noise samples. $\mathbf{R}_{S}\left(N-D_{0}\right)$ is a standard spiked population covariance matrix of rank-1 and $\mathbf{R}_{N}\left(D_{0}\right)$ is Wishart matrix. The largest eigenvalue of $\mathbf{R}_{N}\left(D_{0}\right)$ is negligible compared to the largest eigenvalue of $\mathbf{R}_{S}\left(N-D_{0}\right)$ given a signal identifiability condition is met [6]. It is known that the fluctuation of the largest eigenvalue of a rank-1 spiked population matrix normalized by the noise variance are asymptotically Gaussian $[17,19]$ if the signal identifiability condition is met otherwise its distribution is again a Tracy-Widom of order 2 .

$$
\frac{\left.\lambda_{1}^{T S}\right|_{\mathcal{H}_{0}}}{\sigma_{v}^{2}}=f_{D}\left(x,\left(N-D_{0}\right)\right)
$$

Using the results from (16) and (20), the RLRT decision statistic under null hypothesis can be written as, 


$$
\begin{aligned}
T_{R L R T} \mid \mathcal{H}_{0} & =\frac{\lambda_{1} \mid \mathcal{H}_{0}}{\sigma_{v}^{2}} \\
& =p_{S S}\left|\mathcal{H}_{0} \frac{\left.\lambda_{1}^{S S}\right|_{\mathcal{H}_{0}}}{\sigma_{v}^{2}}+p_{T S}\right|_{\mathcal{H}_{0}} \frac{\left.\lambda_{1}^{T S}\right|_{\mathcal{H}_{0}}}{\sigma_{v}^{2}} \\
& =\left.p_{S S}\right|_{\mathcal{H}_{0}} f_{T W}\left(\frac{t-\mu_{N, K}}{\sigma_{N, K}}\right)+\left.p_{T S}\right|_{\mathcal{H}_{0}} f_{D}\left(x, N-D_{0}\right) .
\end{aligned}
$$

Using the fact that $D_{0}$ is a random variable distributed as in (5), we obtain the final distribution of the decision statistic of RLRT test under null hypothesis as in (12).

We consider now the case when the PU signal is present (hypothesis $\mathcal{H}_{1}$ ). In this case, an error is made if the presence of PU signal is not detected. Under alternate hypothesis $\mathcal{H}_{1}$, the received sample covariance matrix $\left.\mathbf{R}\right|_{\mathcal{H}_{1}}$ can be decomposed as a probabilistic sum of $\left.\mathbf{R}_{S S}\right|_{\mathcal{H}_{1}}$ and $\left.\mathbf{R}_{T S}\right|_{\mathcal{H}_{1}}$.

$$
\left.\mathbf{R}\right|_{\mathcal{H}_{1}}=\left.\left.p_{S S}\right|_{\mathcal{H}_{1}} \mathbf{R}_{S S}\right|_{\mathcal{H}_{1}}+\left.p_{T S}{\mid \mathcal{H}_{1}} \mathbf{R}_{T S}\right|_{\mathcal{H}_{1}}
$$

Since $\left.\mathbf{R}_{S S}\right|_{\mathcal{H}_{1}}$ is a standard spiked population covariance matrix of rank-1, the distribution of the largest eigenvalue normalized by the noise variance in a SS sensing slot under $\mathcal{H}_{1}$ can be approximated as $[17,19]$,

$$
\frac{\left.\lambda_{1}^{S S}\right|_{\mathcal{H}_{1}}}{\sigma_{v}^{2}}=f_{D}(x, N) .
$$

Using the same line of reasoning as in $\mathcal{H}_{0}$, we get,

$$
\frac{\left.\lambda_{1}^{T S}\right|_{\mathcal{H}_{1}}}{\sigma_{v}^{2}}=f_{D}\left(x, D_{1}\right) .
$$

Using (25) and (26), the distribution of the RLRT decision statistic under alternate hypothesis can be written as,

$$
\begin{aligned}
\left.T_{R L R T}\right|_{\mathcal{H}_{1}} & =\frac{\left.\lambda_{1}\right|_{\mathcal{H}_{1}}}{\sigma_{v}^{2}} \\
& =\left.p_{S S}\right|_{\mathcal{H}_{1}} \frac{\left.\lambda_{1}^{S S}\right|_{\mathcal{H}_{1}}}{\sigma_{v}^{2}}+\left.p_{T S}\right|_{\mathcal{H}_{1}} \frac{\left.\lambda_{1}^{T S}\right|_{\mathcal{H}_{1}}}{\sigma_{v}^{2}} \\
& =\left.p_{S S}\right|_{\mathcal{H}_{1}} f_{D}(x, N)+\left.p_{T S}\right|_{\mathcal{H}_{1}} f_{D}\left(x, D_{1}\right) .
\end{aligned}
$$

Incorporating the pmf of $D_{1}$ (derived in (5)) in (29) yields (13).

A. Probability of False Alarm: Given the pdf of the decision statistic in (12), we can compute the false-alarm probability. Under $\mathcal{H}_{0}$, the PU is in free state at the end of the sensing interval, but the decision statistic is erroneously above the threshold $\tau$ and the PU signal is declared present. For defining the probability of false-alarm $P_{F}$ in our case, the following Corollary of Theorem 1 holds. 
Corollary 1. The false-alarm probability of the RLRT test under unknown PU traffic and complex signal space scenario is:

$$
P_{F}=P\left(\left.T_{R L R T}\right|_{\mathcal{H}_{0}} \geq \tau\right) \equiv \int_{\tau}^{+\infty} f_{\left.T_{R L R T}\right|_{H_{0}}}(x) d x .
$$

B. Probability of Detection: Given the pdf of the decision statistic in (13), we can compute the detection probability. Under $\mathcal{H}_{1}$, i.e., the PU is in busy state at the end of the sensing interval. Under this scenario, if the decision statistic is above the threshold, the PU signal is declared present. The following Corollary of Theorem 1 holds for defining the probability of detection $P_{D}$.

Corollary 2. The detection probability of the RLRT test under unknown PU traffic and the complex signal space scenario is:

$$
P_{D}=P\left(\left.T_{R L R T}\right|_{\mathcal{H}_{1}} \geq \tau\right) \equiv \int_{\tau}^{+\infty} f_{\left.T_{R L R T}\right|_{H_{1}}}(x) d x .
$$

\section{Numerical Results and Discussion}

In this section, the effect of PU traffic on the RLRT detection method is analyzed based on the the traffic model developed in Sec. 3. The length of the free and busy periods of the PU traffic are measured in terms of the discrete number of samples where each of them has Geometric distribution with mean parameters $M_{f}$ and $M_{b}$, respectively as described in Sec. 3. Under multiple antenna sensing scenario, the average SNR at the receiver is defined as, $\rho=\frac{\sigma_{s}^{2}\|\boldsymbol{h}\|^{2}}{K \sigma_{v}^{2}}$, where $\|$. denotes the Euclidean norm. The analytical expressions derived in Sec. 4 are validated via numerical simulations.

In Fig. 2, the sensing performance of RLRT under unknown PU traffic is compared with the ideal RLRT performance. It can be well understood that the conventional model with perfect synchronization of the PU-SU sensing slots performs better than the one with unknown PU traffic. In addition, the accuracy of the derived analytical expressions of $P_{F}$ and $P_{D}$ are confirmed where the theoretical formulas are compared against the numerical results obtained by Monte-Carlo simulations. The perfect match of the theoretical and numerical curves validates the derived analytical expressions. The Receiver Operating Characteristics (ROC) performance of RLRT in the considered PU traffic model for different PU traffic parameters is presented in Fig. 2(a). The sensing performance degrades significantly when the mean lengths of busy and free periods are comparable with the length of the sensing interval or in a few multiples of it. However, an improvement in the sensing performance can be seen if the length of the mean parameters $M_{f}$ and $M_{b}$ is increased. We present in Fig. 2(b), the missed detection probability $\left(P_{M d}\right)$ as a function of SNR. From this figure, it is seen that, for a given PU traffic parameters, increasing the SNR improves the sensing performance for certain lower range of SNR. However, in contrast to RLRT sensing performance under known PU traffic, the RLRT sensing performance under unknown PU traffic levels to some point $\left(1>P_{M d}>>0\right)$ above 


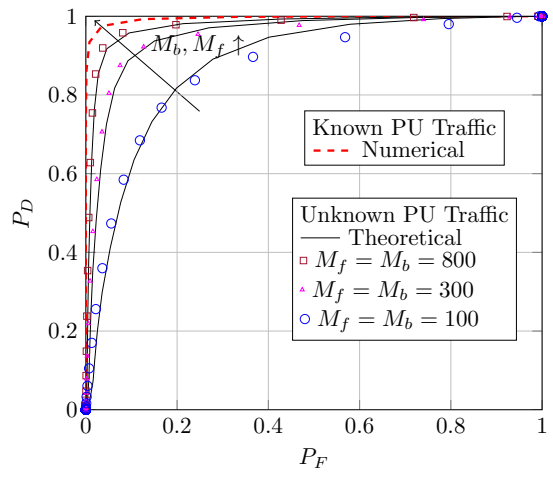

(a) ROC performance, Parameters: $N=50, K=4$ and $\mathrm{SNR}=-6 \mathrm{~dB}$.

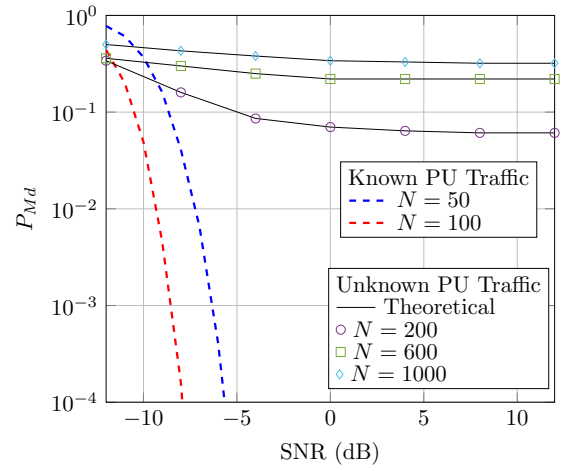

(b) Performance curve (Probability of Missed detection vs. SNR), Parameters: $K=8, P_{F}=0.01$ and $M_{f}=M_{b}=$ 3000 .

Fig. 2: Sensing performance of RLRT under unknown PU traffic.

certain SNR. This is due to the effect of the mixing of the PU signal-plus-noise and only-noise samples in the TS sensing slot.

In Fig. 3, the RLRT sensing performance is plotted as a function of sensing parameters $N$ and $K$. The variation of the sensing performance of RLRT detector for different number of receiving antennas $(K)$ is plotted in Fig. 3(a). It can be observed that, unlike the rapid increase in sensing performance (decrease in missed-detection probability) with the increasing number of receiving antennas under synchronized PU-SU sensing slot scenario, the RLRT sensing performance under unknown PU traffic is almost constant even if we increase the number of antennas. During a TS sensing slot, from each receiving antenna, the received signal samples are the mixture of pure noise samples and the samples with both noise and PU signal. Thus, even if we use multiple antennas, the nature of the received signal doesn't change much which is the reason the sensing performance improvement is suppressed by the unknown PU traffic (more specifically, the TS sensing performance) when the length of the free and busy periods of PU traffic are quite small (a few multiples of the length of the sensing window). Furthermore, we present in Fig. 3(b), the numerical simulation of detection probability $\left(P_{D}\right)$ as a function of sensing window $(N)$. Note that, unlike RLRT detection probability under known PU traffic which monotonically increases indefinitely until ' $P_{D}=1$ ' with increasing length of sensing window, the detection probability of RLRT under unknown PU traffic do not have a monotonic property as a function of the length of the sensing window. 


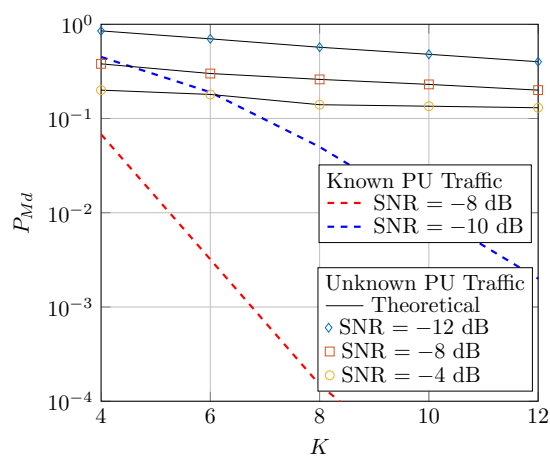

(a) Prob. of Missed Detection vs. the number of receiving antennas $(K)$, Parameters: $N=100, P_{F}=0.01$ and $M_{f}=M_{b}=1500$.

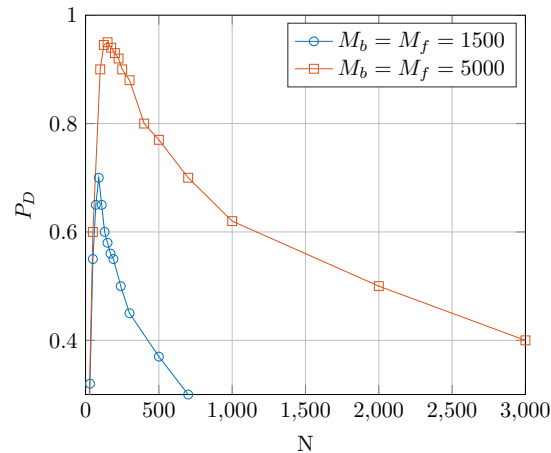

(b) Variation of Detection Probability with increasing $N$, Parameters: $\mathrm{SNR}=$ $-10 \mathrm{~dB}, K=8$ and $P_{F}=0.01$

Fig. 3: RLRT sensing performance comparison for different sensing parameters $(N$ and $K)$.

\section{Conclusion}

In this paper, the effect of PU traffic on the performance of largest eigenvalue based detection technique (RLRT) has been studied under the complex domain of PU signal, noise and channel. A realistic and simple PU traffic model has been considered which is based only on the discrete time distribution of PU free and busy periods. Moreover, an analytical evaluation of the spectrum sensing performance under the considered scenario has been carried out. It has been observed that the sensing performance of RLRT is no more monotonically increasing with the increase in the length of the sensing duration and also with SNR which contrasts with the common property of the spectrum sensing techniques under known PU traffc scenario. Finally, it has been observed that the performance gain due to multiple antennas in the sensing unit is significantly suppressed by the effect of the PU traffic when the frequencies of the PU traffic transitions are more frequent.

\section{Acknowledgment}

This work was partially supported by FNR, Luxembourg under the CORE projects "SeMIGod" and "SATSENT" and also by the EC under FP7 NEWCOM\# (Grant agreement no. 318306).

\section{References}

1. Haykin, S.: "Cognitive radio: Brain-empowered wireless communications", IEEE Journal on Selected Areas in Commun., vol. 23, no. 2, pp. 201-220, Feb. 2005. 
2. Axell, E., Leus, G., Larsson, E. G., and Poor, H. V.: "Spectrum sensing for cognitive radio:State-of-the-art and recent advances," IEEE Signal Process. Mag., vol. 29, no. 3, pp. 101-116, May 2012.

3. Sharma, S. K., Bogale, T. E., Chatzinotas, S., Ottersten, B., Le L. B., and Wang, X.: "Cognitive Radio Techniques under Practical Imperfections: A Survey", IEEE Commun. Surveys Tutorials, July, 2015.

4. Consultative Committee for Space Data Systems, "TC synchronization and channel coding", CCSDS 231.0-B-2 Blue Book, Sep. 2010.

5. Zorzi, M., Gluhak, A., Lange, S., Bassi, A.: "From today's INTRAnet of things to a future INTERnet of things: a wireless- and mobility-related view", IEEE Wireless Commun. Mag., vol. 17, no. 6, pp.44-51, Dec. 2010.

6. Penna, F. and Garello, R.: "Detection of Discontinuous Signals for Cognitive Radio Applications", IET Commun., vol. 5, no. 10, pp. 1453-1461, Jan. 2011.

7. Palit, R., Naik, K., Singh, A.: "Anatomy of WiFi access traffic of smart-phones and implications for energy saving techniques", Intl. J. of Energy, Inform. and Commun., vol. 3, Feb. 2012.

8. Ghosh, A., Jana, R., Ramaswami, V., Rowland, J., Shankaranarayanan, N., K.: "Modeling and characterization of large-scale Wi-Fi traffic in public hot-spots", IEEE Proc. INFOCOM, pp. 2921-2929, April 2011.

9. MacDonald, S. L. and Popescu, D. C.: "Impact of primary user activity on the performance of energy-based spectrum sensing in cognitive radio systems", IEEE Proc. GLOBECOM, pp. 3224-3228, Dec. 2013.

10. Wang, T., Chen, Y., Hines, E. and Zhao, B.: "Analysis of effect of primary user traffic on spectrum sensing performance", in Proc. International Conf. Commun. Netw. China, pp. 1-5. Aug. 2005.

11. Wu, J. Y., Huang, P. H., Wang, T. Y. and Wong, V. W. S.: "Energy detection based spectrum sensing with random arrival and departure of primary user's signal", IEEE Proc. GLOBECOM, pp. 380-384, Dec. 2013.

12. Tang, L., Chen, Y., Hines, E. L. and Alouini, M. S.: "Effect of primary user traffic on sensing-throughput tradeoff for cognitive radios', IEEE Trans. Wireless Commun., vol. 10, no. 4, pp. 1063-1068, Apr. 2011.

13. Pradhan, H., Kalamkar, A. S. and Banerjee, A.: "Sensing-throughput tradeoff in cognitive radio with random arrivals and departures of multiple primary users", IEEE Commun. Letters, vol.19, no.3, pp.415-418, March 2015.

14. Hwei P. Hsu, "Theory and Problems of Probability, Random Variables, and Random Processes", SCHAUM'S OUTLINE SERIES McGRAW-HILL, 1996.

15. Roy, S. N.: "On a heuristic method of test construction and its use in multivariate analysis", Ann. Math. Stat., pp. 220238, 1953.

16. Y. H. Zeng, Y. C. Liang, "Eigenvalue based spectrum sensing algorithms for cognitive radio", IEEE Trans. Commun., vol. 57, no. 6, pp. 1784-1793, 2009

17. Dhakal, P., Riviello, D., Penna, F., and Garello, R.: "Impact of noise estimation on energy detection and eigenvalue based spectrum sensing algorithms", IEEE Proc. ICC, pp. 1367-1372, June, 2014.

18. Bianchi, P., Najim, J., Alfano, G., Debbah, M.: "Asymptotics of eigenbased collaborative sensing". Proc. IEEE Inform. Theory Workshop (ITW 2009), Oct. 2009

19. Penna, F., Garello, R., Spirito, M.A.: "Probability of missed detection in eigenvalue ratio spectrum sensing". Fifth IEEE Int. Conf. on WiMob, Oct. 2009.

20. Bai, Z.D.: "Methodologies in spectral analysis of large-dimensional random matrices, a review", Stat. Sin., vol. 9 , pp. 611-677, 1999. 